\title{
Cross-comparison of cardiac output trending accuracy of LiDCO, PiCCO, FloTrac and pulmonary artery catheters
}

Mehrnaz Hadian ${ }^{1,3}$, Hyung Kook Kim', Donald A Severyn², Michael R Pinsky ${ }^{1 *}$

\begin{abstract}
Introduction: Although less invasive than pulmonary artery catheters (PACs), arterial pulse pressure analysis techniques for estimating cardiac output (CO) have not been simultaneously compared to PAC bolus thermodilution CO (COtd) or continuous CO (CCO) devices.

Methods: We compared the accuracy, bias and trending ability of $\mathrm{LiDCO}^{\mathrm{TM}}$, PiCCO ${ }^{\mathrm{TM}}$ and FloTrac ${ }^{\mathrm{TM}}$ with PACs (COtd, CCO) to simultaneously track CO in a prospective observational study in 17 postoperative cardiac surgery patients for the first 4 hours following intensive care unit admission. Fifty-five paired simultaneous quadruple CO measurements were made before and after therapeutic interventions (volume, vasopressor/dilator, and inotrope).

Results: Mean CO values for PAC, LiDCO, PiCCO and FloTrac were similar (5.6 $\pm 1.5,5.4 \pm 1.6,5.4 \pm 1.5$ and $6.1 \pm$ $1.9 \mathrm{~L} / \mathrm{min}$, respectively). The mean CO bias by each paired method was -0.18 (PAC-LiDCO), 0.24 (PAC-PiCCO), -0.43 (PAC-FloTrac), 0.06 (LiDCO-PiCCO), -0.63 (LiDCO-FloTrac) and -0.67 L/min (PiCCO-FloTrac), with limits of agreement (1.96 standard deviation, 95\% confidence interval) of $\pm 1.56, \pm 2.22, \pm 3.37, \pm 2.03, \pm 2.97$ and $\pm 3.44 \mathrm{~L} / \mathrm{min}$, respectively. The instantaneous directional changes between any paired CO measurements displayed 74\% (PACLiDCO), 72\% (PAC-PiCCO), 59\% (PAC-FloTrac), 70\% (LiDCO-PiCCO), 71\% (LiDCO-FloTrac) and 63\% (PiCCO-FloTrac) concordance, but poor correlation $\left(r^{2}=0.36,0.11,0.08,0.20,0.23\right.$ and 0.11 , respectively). For mean $\mathrm{CO}<5 \mathrm{~L} / \mathrm{min}$ measured by each paired devices, the bias decreased slightly.

Conclusions: Although PAC (COTD/CCO), FloTrac, LiDCO and PiCCO display similar mean CO values, they often trend differently in response to therapy and show different interdevice agreement. In the clinically relevant low CO range $(<5 \mathrm{~L} / \mathrm{min})$, agreement improved slightly. Thus, utility and validation studies using only one CO device may potentially not be extrapolated to equivalency of using another similar device.
\end{abstract}

\section{Introduction}

Although the pulmonary arterial catheter (PAC) measures cardiac output $(\mathrm{CO})$ easily at the bedside in critically ill patients [1-3], the recent trend in intensive care unit (ICU) monitoring is toward minimally invasive methods [4-8]. Arterial pulse contour and pulse power analyses have emerged as less invasive alternatives to PAC-derived CO measures $[9,10]$. The accuracy of these devices for PAC-derived $\mathrm{CO}$ measures has not been systematically compared in response to therapies other than volume resuscitation $[11,12]$. These devices use

\footnotetext{
* Correspondence: pinskymr@upmc.edu

'Department of Critical Care Medicine, University of Pittsburgh Medical

Center, 230 Lothrop Street, Pittsburgh, PA 15261, USA

Full list of author information is available at the end of the article
}

different calibration schema and model the transfer of arterial pulse pressure to stroke volume differently. Thus, their cross-correlations may not be assumed to be similar. The LiDCO Plus ${ }^{\mathrm{Th}}$ (LiDCO Ltd, London, UK) uses a transthoracic lithium dilution estimate of $\mathrm{CO}$ for calibration, whereas the PiCCO Plus ${ }^{\mathrm{Tu}}$ (Pulsion Ltd, Munich, Germany) uses a transthoracic thermodilution approach to compensate for interindividual differences in arterial compliance [13-15]. The FloTrac ${ }^{\text {tw }}$ calculates $\mathrm{CO}$ from the pulse contour using a proprietary algorithm and patient-specific demographic data [16] with, however, inconsistent reports of accuracy [17-20].

Although all devices have been compared individually to PAC-derived estimates of $\mathrm{CO}$, none have been compared to each other [21]. Oxygen delivery $\left(\mathrm{DO}_{2}\right)$
() Biomed Central

C 2010 Hadian et al.; licensee BioMed Central Ltd. This is an open access article distributed under the terms of the Creative Commons Attribution License (http://creativecommons.org/licenses/by/2.0), which permits unrestricted use, distribution, and reproduction in any medium, provided the original work is properly cited. 
targeted resuscitation algorithms may improve outcomes in selected patient groups [22]. Thus, knowing the degree to which different systems co-vary is important if one is to use these outcome studies in a general fashion to define the utility of all minimally invasive monitoring systems. Accordingly, in this study, we cross-compared the $\mathrm{CO}$ values and their changes in a critically ill patient cohort in whom active changes in blood volume, vasomotor tone and contractility were induced by specific therapies. We compared three pulse contour devices (LiDCO Plus, PiCCO Plus and FloTrac) (Edwards Lifesciences, Irvine, CA, USA) and two PAC thermodilution techniques: $\mathrm{CO}$ by thermodilution (COtd) and continuous cardiac output (CCO) in postoperative cardiac surgery patients during the first 4 postoperative ICU hours when most of the aggressive treatments occurred. To minimize initial $\mathrm{CO}$ differences, we calibrated the $\mathrm{PiCCO}$ and LiDCO devices using the initial PAC CO values, whereas the FloTrac did not allow external calibration.

\section{Materials and methods}

The study was approved by our Institutional Review Board, and all patients provided signed informed consent. Twenty postcardiac surgery patients (age range, 54 to $82 \mathrm{yr}$ ) were studied. Additional inclusion criteria were the presence of both an arterial catheter and PAC (Edwards LifeSciences, Irvine, CA, USA) (either $\mathrm{CO}_{\mathrm{TD}}$ or $\mathrm{CCO}$ ). Exclusion criteria were evidence of cardiac contractility dysfunction (ejection fraction $<45 \%$ by intraoperative echocardiography), pregnancy, having pacemaker or automated implantable cardioverter-defibrillator, persistent arrhythmias, heart and/or lung transplant, severe valvular (mitral, aortic, pulmonic or tricuspid) stenosis or insufficiency after surgery, intraaortic balloon pump or other mechanical cardiac support.

Patients were admitted to the ICU on assist control ventilatory mode with $12 / \mathrm{min}$ respiratory rate (no patient had a spontaneous respiration $>16 / \mathrm{min}$ ) and 6 $\mathrm{ml} / \mathrm{kg}$ tidal volume, inspiratory-to-exporatory (I/E) time of $1: 2$ and $5 \mathrm{~cm} \mathrm{H}_{2} \mathrm{O}$ positive end-expiratory pressure. Fentanyl $(25-50 \mu \mathrm{g})$ was given as needed by nursing staff if the patient appeared to have pain or discomfort.

\section{FloTrac ${ }^{\mathrm{TM}}$ and PAC}

The FloTrac ${ }^{\mathrm{Tn}}$ pulse contour device (Vigileo ${ }^{\mathrm{mm}}$, Edwards LifeSciences, Irvine, CA, USA) was attached to the existing arterial cannula, and its sensor was attached to the processing or display unit to read CO. The patient's demographic data (height, weight, age, and gender) were entered into the device as recommended by the manufacturer. FloTrac $\mathrm{CO}$ is reported as an averaged value over 20 seconds using a proprietary algorithm [23]. All continuous $\mathrm{CO}$ measurements were collected from the Vigileo $^{\text {tw }}$ monitor and input into a WinDaq data acquisition system (WINDAQ V 1.26, Dataq Instruments Inc., Akron, $\mathrm{OH}$ ) as previously described [24].

Either a $\mathrm{CO}_{\mathrm{TD}}$ or a $\mathrm{CCO}$ was measured by a standard PAC attached to Vigilance ${ }^{\mathrm{Tu}}$ monitor (Edwards LifeSciences, Irvine, CA). If a non-CCO PAC was present, then $\mathrm{CO}$ measurements were taken upon patient arrival to the ICU and then after each therapeutic intervention as described below. $\mathrm{CO}_{\mathrm{TD}}$ was taken as the mean of at least three $10-\mathrm{ml} 5^{\circ} \mathrm{C} 0.9 \mathrm{~N} \mathrm{NaCl}$ bolus injections random to the respiratory cycle. The accuracy and acceptability of each thermal decay curve was judged visually on the attached ICU monitor. If CCO PAC was present, then all CCO data based on STAT values were continuously collected until end of the study using the WinDaq data acquisition.

\section{LiDCO plus $^{\mathrm{Tm}}$ and PiCCO plus ${ }^{\mathrm{Tm}}$}

Arterial wave form data was collected using the WinDaq data acquisition system as previously described [24]. These waveforms were then reinjected into both the LiDCO plus $^{\text {Tix }}$ and PiCCO plus ${ }^{\mathrm{Tw}}$ devices offline to calculate CO. To minimize differences due to initial calibration variance, both the LiDCO plus ${ }^{\mathrm{Tm}}$ and PiCCO plus ${ }^{\mathrm{mm}}$ devices had their initial $\mathrm{CO}$ values taken from the simultaneous PACderived $\mathrm{CO}$ values at time 0 as recommended by the manufacturers, after which time neither device was recalibrated. All continuous LiDCO and PiCCO CO measurements were collected in a data acquisition system installed internally in the device. The clocks on the all data acquisition systems were matched. All the $\mathrm{CO}_{\mathrm{TD}}$ measurements were taken by one investigator $(\mathrm{MH})$.

\section{Protocol}

We compared the mean paired $\mathrm{CO}$ values $30 \mathrm{~s}$ before and 1-2 min after ending a volume challenge and after heart rate and blood pressure stabilized $(<5 \%$ variation over $30 \mathrm{~s}$ ) following changes in vasoactive and inotropic therapy. We made no attempt to alter the usual care of the patients. The FloTrac data were blinded to the primary care physicians. All paired event data were downloaded in a common Microsoft Excel (Microsoft Corp., Redmond, WA, USA) spreadsheet for statistical analysis.

\section{Statistical analysis}

We performed analysis of variance for comparison of mean baseline CO between the three devices. A post hoc Student's paired $t$-test was used to compare groups when significance was identified. $P<0.05$ was considered significant. We performed Bland-Altman analysis for paired devices PAC-LiDCO, PAC-PiCCO, PAC-FloTrac, LiDCOPiCCO, LiDCO-FloTrac and PiCCO-FloTrac. Bias was defined as the mean difference between $\mathrm{CO}$ measurements 
by each set of paired devices. The upper and lower limits of agreement were defined as \pm 1.96 standard deviation (SD) of the bias. The percentage error was calculated as limits of agreement divided by the mean $\mathrm{CO}[25,26]$. Bias, limits of agreement and percentage error were calculated for the entire data set for each set of paired devices and then separately for $\mathrm{CO}_{\mathrm{TD}}$ and $\mathrm{CCO}$. We also performed two additional Bland-Altman analyses. We selectively compared limits of agreement and bias of $\mathrm{CO}$ values $<5 \mathrm{~L} /$ min to ascertain whether any observed bias was selectively due to higher flow rates, which would have less clinical relevance. Since there is no reference $\mathrm{CO}$ measure, we also created a pooled $\mathrm{CO}$ measure as the mean of all the devices' $\mathrm{CO}$ values at one point ( $Z$-statistic) and performed a Bland-Altman analysis of each device against this mean of all devices. For this analysis, we pooled the PAC COtd and CCO values into one variable. Since directional changes in $\mathrm{CO}$ are important in assessing response to therapy, the degree of concordance was defined as the percentage of the total number of events when paired devices showed the same directional change in $\mathrm{CO}$ (greater than $\pm 0.5 \mathrm{~L} / \mathrm{min}$ ) divided by the total number of events using a Pearson product-moment correlation coefficient analysis. We assumed that all paired $\mathrm{CO}$ data that varied by $<0.5 \mathrm{~L} / \mathrm{min}$ reflected no change and then calculated the percentage of paired data points when both devices reported no change or a change of $>0.5 \mathrm{~L} / \mathrm{min}$ in the same direction. We also calculated the correlation of the dynamic changes in these paired values using simple linear correlation analysis.

\section{Results}

Table 1 reports patient demographics. Simultaneous CO measurements for all four devices in 17 patients were

Table 1 Patient demographic data ${ }^{a}$

\begin{tabular}{lc}
\hline Age $(\mathrm{yr})$ & $73 \pm 9$ \\
Gender (M/F) & $11 / 6$ \\
LVEF (\%) & $52 \pm 8$ \\
Type of PAC (COTD/CCO) & $10 / 7$ \\
Arterial catheter site (femoral/radial) & $9 / 8$ \\
Type of operation & Number \\
$\quad$ CABG & 8 \\
$\quad$ AVR & 2 \\
$\quad$ MVR & 1 \\
$\quad$ AVR + MVR & 1 \\
$\quad$ TABG + AVR & 1 \\
CABG + AVR +TAAR & 3 \\
\hline
\end{tabular}

${ }^{a}$ Data are presented as means $\pm S D$. LVEF, left ventricular ejection fraction; PAC, pulmonary artery catheter; $\mathrm{CO}_{\mathrm{TD}} / \mathrm{CCO}$, intermittent bolus thermodilution/ continuous cardiac output; CABG, coronary artery bypass grafting; AVR/MVR/ TVR, aortic/mitral/tricuspid valve repair or replacement; TAAR, thoracic aortic aneurysm repair, $n=17$. taken. Two patients were excluded from analysis because of arrhythmias and another was excluded because the arterial pressure waveforms recorded were unusable for the PiCCO device. Table 2 reports CO by device and treatment intervention characteristics. Although mean $\mathrm{CO}$ values for PAC, LiDCO, PiCCO and FloTrac were not different (5.6 $\pm 1.5,5.4 \pm 1.6,5.4 \pm 1.5$ and $6.1 \pm 1.9 \mathrm{~L} / \mathrm{min}$, respectively), mean FloTrac $\mathrm{CO}$ values were slightly higher than others, approaching statistical significance between PAC, LiDCO and PiCCO $(P=0.095,0.120$ and 0.078 , respectively).

The mean $\mathrm{CO}$ bias between each paired method was -0.18 (PAC-LiDCO), 0.24 (PAC-PiCCO), -0.43 (PACFloTrac), 0.06 (LiDCO-PiCCO), -0.63 (LiDCO-FloTrac) and $-0.67 \mathrm{~L} / \mathrm{min}$ (PiCCO-FloTrac), with limits of agreement $(1.96 \mathrm{SD}, 95 \% \mathrm{CI})$ of $\pm 1.56, \pm 2.22, \pm 3.37, \pm 2.03$, \pm 2.97 and $\pm 3.44 \mathrm{~L} / \mathrm{min}$, respectively (Figure 1 ). The percentage error for each set of paired devices was $29 \%$, $41 \%, 59 \%, 39 \%, 53 \%$ and $61 \%$, respectively.

Since $\mathrm{CO}$ accuracy may be clinically more important at low $\mathrm{CO}$ values, we analyzed the agreement among estimates of $\mathrm{CO}$ for mean values $\leqq 5 \mathrm{~L} / \mathrm{min}$. For CO values $\leqq 5 \mathrm{~L} / \mathrm{min}$, bias and limits of agreement were $-0.17 \pm 1.58$ (PAC-LiDCO), $0.27 \pm 1.84$ (PAC-PiCCO),

\section{Table 2 Mean cardiac output measurements ${ }^{a}$}

\begin{tabular}{|c|c|}
\hline \multicolumn{2}{|l|}{ Baseline $\mathrm{CO}(\mathrm{L} / \mathrm{min}), n=17$ using both $\mathrm{CO}_{\mathrm{TD}}$ and $\mathrm{CCO}$} \\
\hline PAC $\left(\mathrm{CO}_{\mathrm{TD}} / \mathrm{CCO}\right)$ & $5.6 \pm 1.5$ \\
\hline LiDCO Plus & $5.4 \pm 1.6$ \\
\hline PiCCO & $5.4 \pm 1.5$ \\
\hline FloTrac/Nigileo & $6.1 \pm 1.9$ \\
\hline \multicolumn{2}{|l|}{ Baseline $\mathrm{CO}(\mathrm{L} / \mathrm{min}), n=10$ using $\mathrm{CO}_{\mathrm{TD}}$} \\
\hline $\mathrm{CO}_{\mathrm{TD}} \mathrm{PAC}$ & $6.0 \pm 1.3$ \\
\hline LiDCO Plus & $5.9 \pm 1.3$ \\
\hline $\mathrm{PiCCO}$ & $6.0 \pm 1.3$ \\
\hline FloTracNigileo & $6.9 \pm 1.5$ \\
\hline \multicolumn{2}{|l|}{ Baseline $\mathrm{CO}(\mathrm{L} / \mathrm{min}), n=7$ using $\mathrm{CCO}$} \\
\hline CCO PAC & $4.8 \pm 1.4$ \\
\hline LiDCO Plus & $4.5 \pm 1.8$ \\
\hline PiCCO & $4.3 \pm 1.5$ \\
\hline FloTracNigileo & $4.8 \pm 1.7$ \\
\hline Therapeutic interventions & Number \\
\hline $\begin{array}{l}\text { Vasodilator (any } \Delta>0.1 \mu \mathrm{g} / \mathrm{kg} / \mathrm{min} \text { in nitroprusside } \\
\text { infusion) }\end{array}$ & 34 \\
\hline $\begin{array}{l}\text { Vasoconstrictor (any } \Delta>0.01 \mu \mathrm{g} / \mathrm{kg} / \mathrm{min} \text { in } \\
\text { norepinephrine or phenylephrine infusion) }\end{array}$ & 8 \\
\hline $\begin{array}{l}\text { Volume challenge (any volume }>250 \mathrm{cc} \text { of PRBC, FFP, } \\
\text { platelets or } 0.9 \% \text { saline given over }<30 \mathrm{~min} \text { ) }\end{array}$ & 8 \\
\hline $\begin{array}{l}\text { Inotrope (any } \Delta>0.01 \mu \mathrm{g} / \mathrm{kg} / \mathrm{min} \text { in epinephrine or }> \\
1 \mu \mathrm{g} / \mathrm{kg} / \mathrm{min} \text { in dopamine or dobutamine infusion) }\end{array}$ & 10 \\
\hline $\begin{array}{l}\text { Combination of any two or more interventions } \\
\text { simultaneously }\end{array}$ & 66 \\
\hline
\end{tabular}

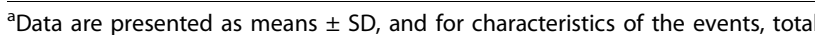
number. PAC, pulmonary artery catheter; $\mathrm{CO}_{\mathrm{TD}} / \mathrm{CCO}$, intermittent bolus/ continuous cardiac output; $\triangle$, change; PRBC, packed red blood cells; FFP, fresh frozen plasma. 

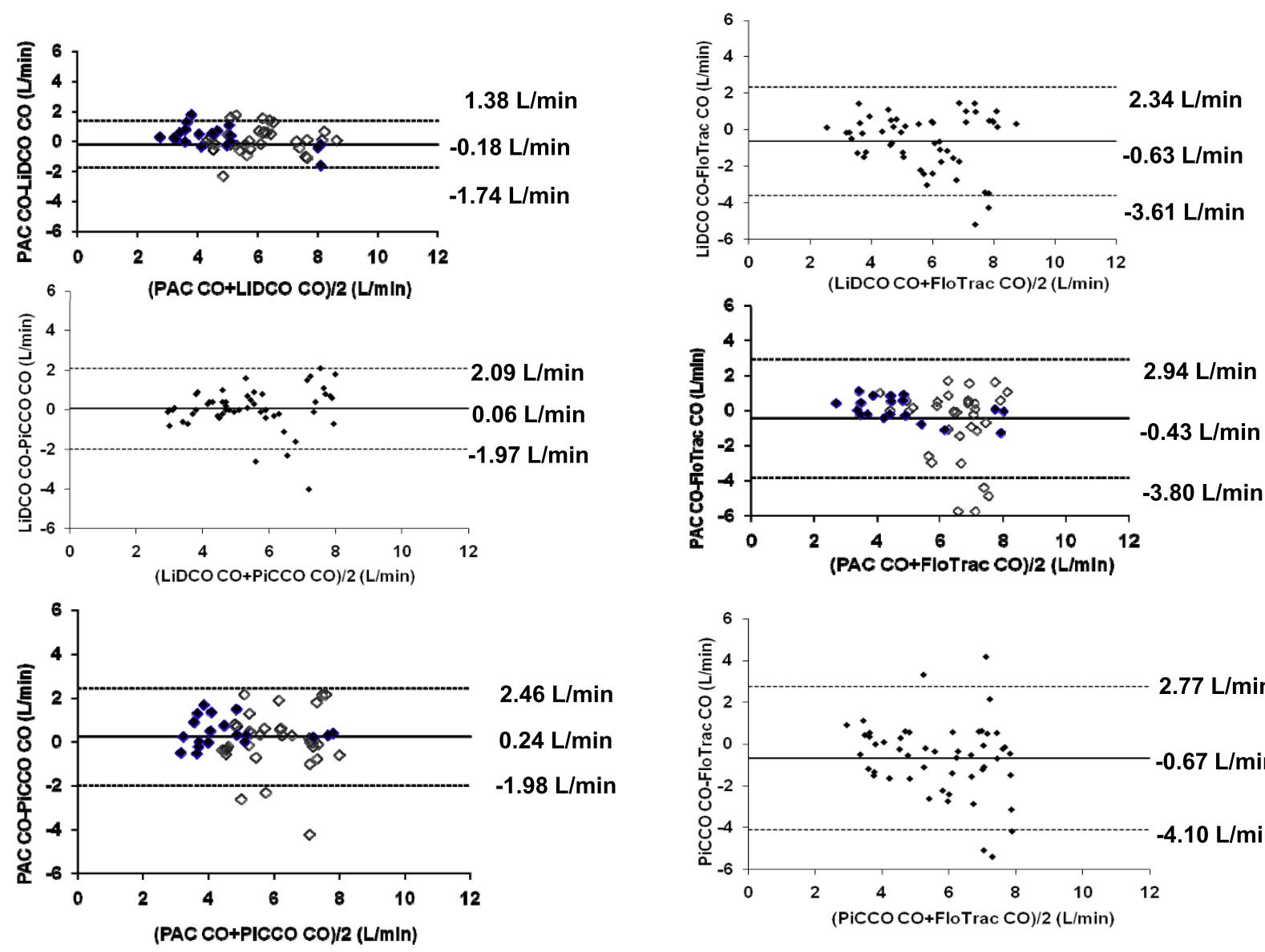

2.46 L/min

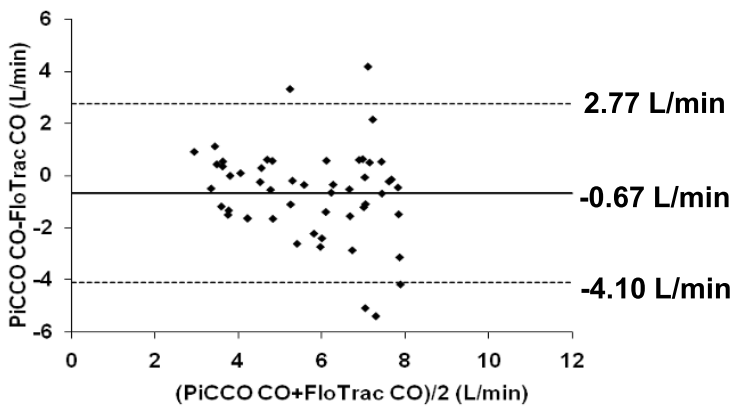

Figure 1 Bland-Altman analysis of each set of paired devices' cardiac output (CO). Solid line, mean difference (bias); dotted lines, limit of agreement (bias \pm 1.96 standard deviation (SD)).

$0.30 \pm 1.00$ (PAC-FloTrac), $0.04 \pm 0.91(\mathrm{LiDCO}-\mathrm{PiCCO})$ $-0.10 \pm 1.56(\mathrm{LiDCO}-$ FloTrac) and $-0.27 \pm 1.86 \mathrm{~L} / \mathrm{min}$ (PiCCO-FloTrac) (Figure 2).

The mean $\mathrm{CO}$ bias between each device and the pooled group $\mathrm{CO}$ values, noting individual device variance from the group mean, was -0.2 (LiDCO), 0.4 (FloTrac), -0.2 (PiCCO) and $0.0 \mathrm{~L} / \mathrm{min}$ (PAC), with limits of agreement $(1.96 \mathrm{SD}, 95 \% \mathrm{CI})$ of $\pm 1.2 \pm 2.4 \pm 1.6$ and \pm 1.4 , respectively (Figure 3 ).

\section{PAC COtd vs. CCO as reference points}

The bias and limits of agreement for each paired method in subgroup analyses of patients with either $\mathrm{CO}_{\mathrm{TD}}$ or CCO PAC are shown in Figure 4. The bias and limits of agreement for LiDCO with CCO $(-0.31 \pm$ $1.41 \mathrm{~L} / \mathrm{min}), \mathrm{PiCCO}$ with CCO $(0.49 \pm 1.30 \mathrm{~L} / \mathrm{min})$ and FloTrac with CCO $(0.05 \pm 1.30 \mathrm{~L} / \mathrm{min})$ were different from that of the three devices with $\mathrm{CO}_{\mathrm{TD}} \mathrm{PAC}$ $(-0.10 \pm 1.64,0.09 \pm 2.58$ and $-0.72 \pm 4.09 \mathrm{~L} / \mathrm{min}$, respectively).
The directional changes between any two paired $\mathrm{CO}$ measurements from before and after each intervention displayed 74\% (PAC-LiDCO), 72\% (PAC-PiCCO), 59\% (PAC-FloTrac), 70\% (LiDCO-PiCCO), 71\% (LiDCO-FloTrac) and 63\% (PiCCO-FloTrac) concordance but poor correlation $\left(r^{2}=0.36, P<0.0001 ; r^{2}=0.11, P=0.025\right.$; $r^{2}=0.08, P=0.079 ; r^{2}=0.20, P=0.002 ; r^{2}=0.23, P=$ 0.001 ; and $r^{2}=0.11, P=0.033$, respectively) (Figure 5).

\section{Discussion}

$\mathrm{DO}_{2}$-targeted resuscitation protocols reduce both length of stay and infectious complications in high-risk surgical patients $[27,28]$. Several minimally invasive monitoring devices have been used to realize these benefits. Our study demonstrates that the three commercially available $\mathrm{CO}$ monitoring devices report similar mean $\mathrm{CO}$ values, but dynamic trends among these devices over clinically relevant $\mathrm{CO}$ changes are not consistent. Thus, in the presence of no contradictory findings, one must use monitors specifically used in a proven effective treatment 


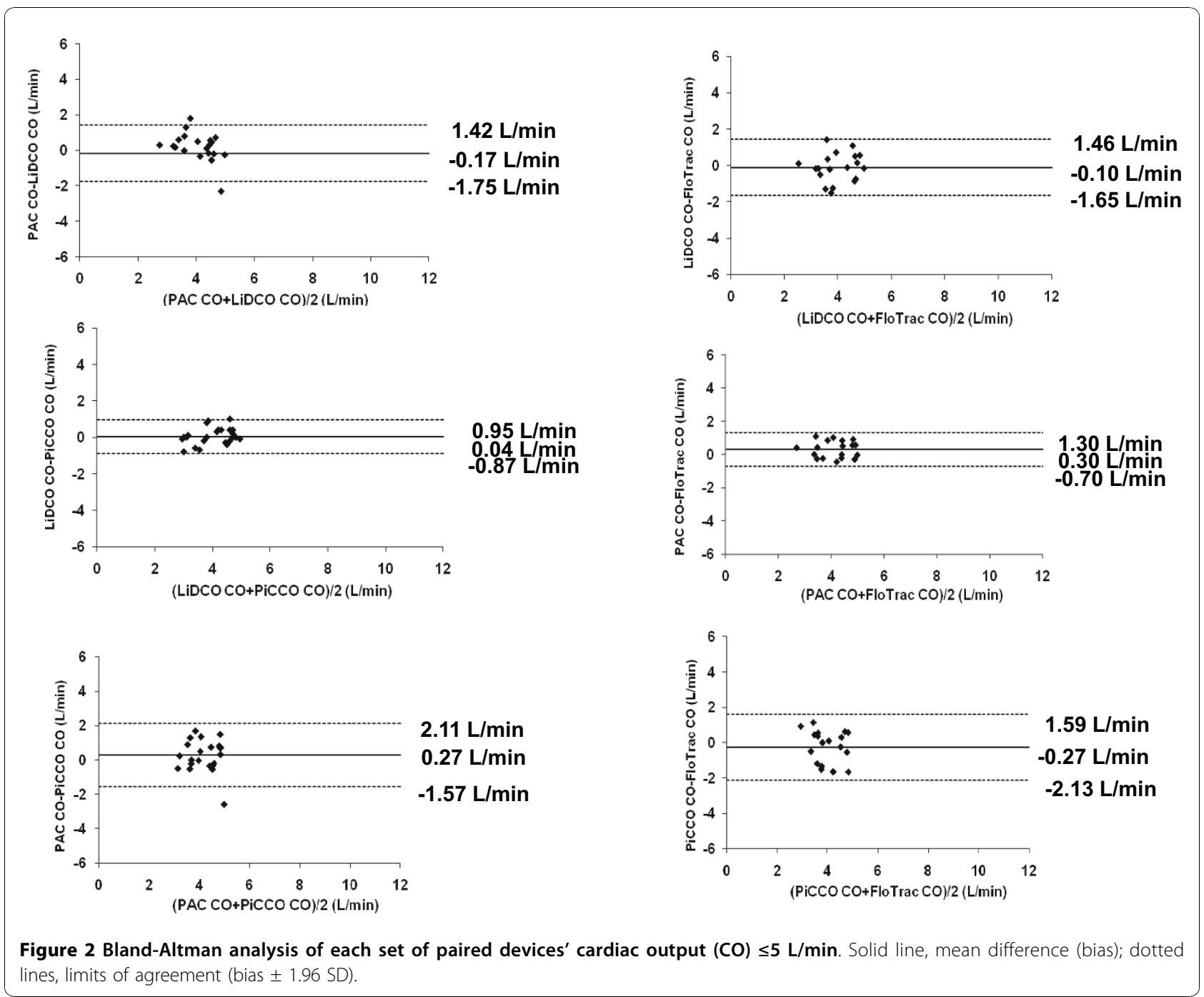

protocol to ensure the utility of that treatment. Within this context, PAC, LiDCO plus $^{\mathrm{Tm}}$ and FloTrac postoptimization protocols have been shown to improve patientcentered outcome $[27,29,30]$. Surprisingly, no comparable PAC data-specific clinical trials have been reported. We are unable to comment on the ability of FloTrac ${ }^{\mathrm{Tm}}$ - or PiCCO plus ${ }^{\mathrm{Tm}}$-guided therapy to improve outcome because they have not been studied in this context. However, on the basis of our analysis of 55 quadruple measures and the three recent clinical trials [18-21,31], it is doubtful that their performance, using the present proprietary iterations, will be interchangeable with PAC or result in any better outcomes than were observed using the LiDCO plus ${ }^{\mathrm{Ta}} \mathrm{CO}$ estimates to target $\mathrm{DO}_{2}$ levels.

This clinical study is unique for two specific reasons. First, we studied three commercially available pulse contour-pulse power analysis devices that report continuous $\mathrm{CO}$ measures and compared them to each other and to two types of PAC CO estimates: COtd or CCO. Since none of these devices is a "gold standard," the three pulse contour devices were compared to each other and to the PAC as equal devices. Our comparisons show that LiDCO plus ${ }^{\mathrm{TM}}$ and PAC have greater agreement with each other than do either PiCCO plus ${ }^{\mathrm{Tm}}$ or FloTrac ${ }^{\mathrm{Tm}}$ with PAC. Furthermore, the limits of agreement between LiDCO plus ${ }^{\mathrm{TM}}$ and PAC are within the boundaries of the Critchey-Critchey criteria [25], whereas those of PiCCO plus $^{\mathrm{TM}}$ or FloTrac and PAC exceed those criteria. This close correlation also agrees with our previous data during open heart surgery, wherein we documented that the LiDCO plus ${ }^{\mathrm{Tm}}$ estimates of stroke volume accurately trend actual left ventricular stroke volume measures during rapid and dynamic changes in $\mathrm{CO}$ when aortic flow was accurately measured in humans using an electromagnetic flow probe placed around the ascending aorta [32]. These levels of agreement difference persist when all devices are compared to a mean pooled $\mathrm{CO}$ value of the group as opposed to each other separately 

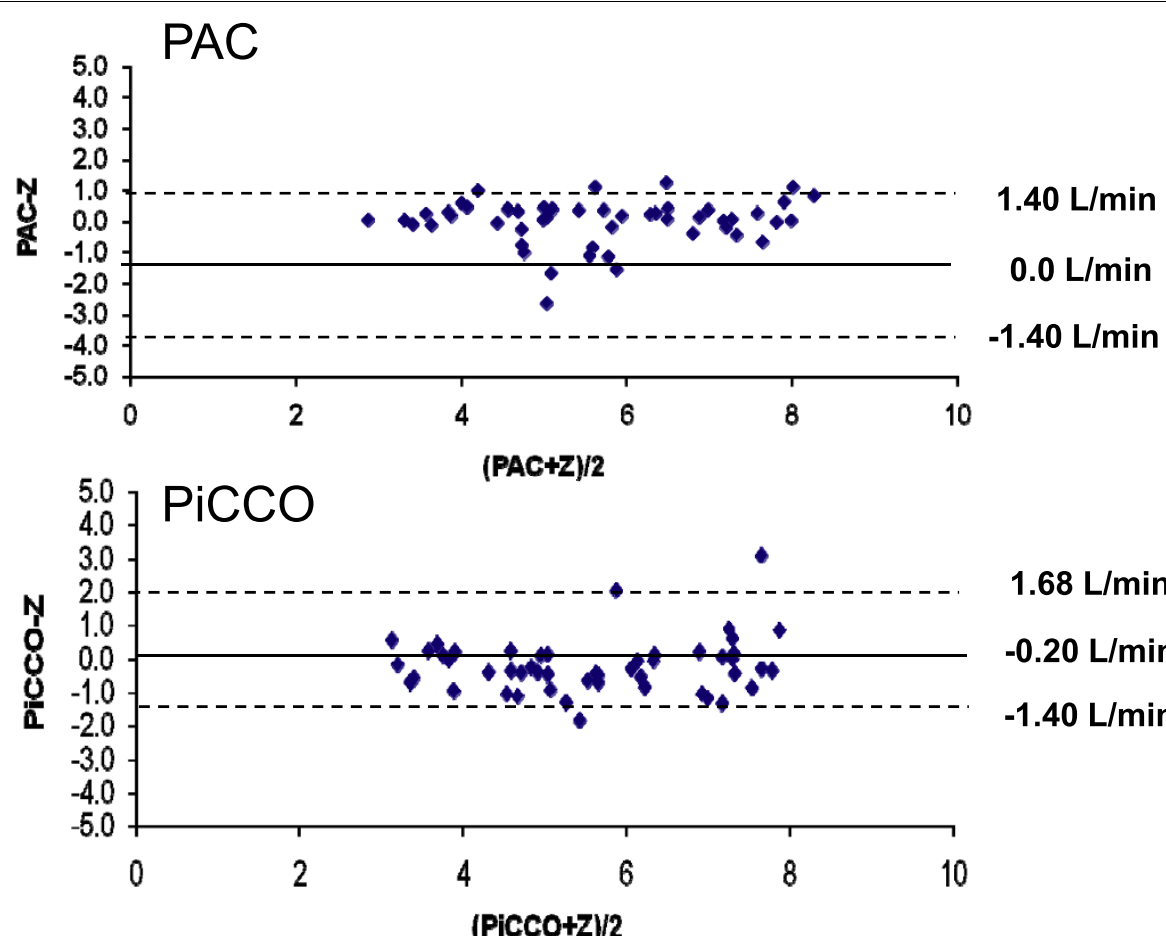

\section{$1.68 \mathrm{~L} / \mathrm{min}$}

\section{$-0.20 \mathrm{~L} / \mathrm{min}$}

$-1.40 \mathrm{~L} / \mathrm{min}$

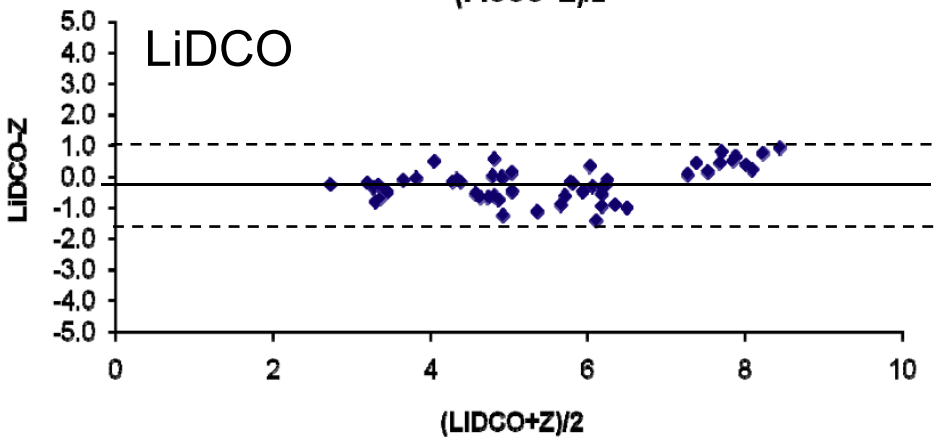

\section{$1.00 \mathrm{~L} / \mathrm{min}$ \\ $-0.20 \mathrm{~L} / \mathrm{min}$ \\ $-1.40 \mathrm{~L} / \mathrm{min}$}

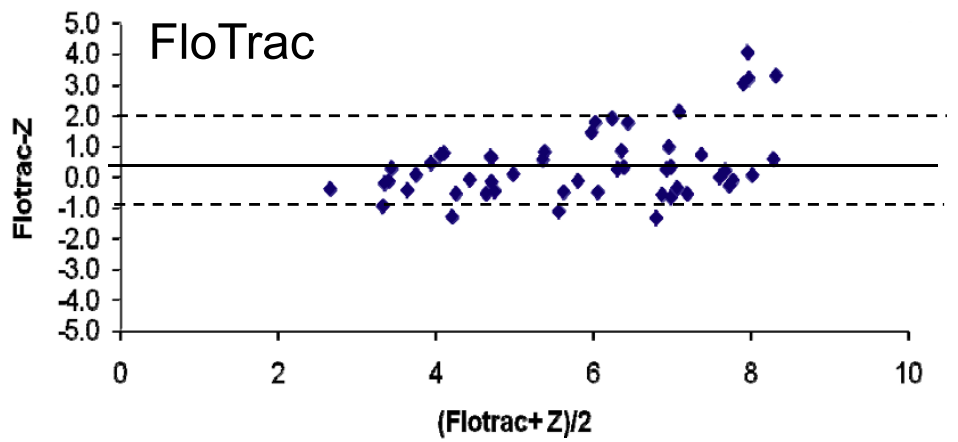

\section{$1.80 \mathrm{~L} / \mathrm{min}$}

$0.40 \mathrm{~L} / \mathrm{min}$

$-1.00 \mathrm{~L} / \mathrm{min}$

Figure 3 Bland-Altman analysis of each device against the mean of all devices across all patients, wherein pulmonary arterial catheter (PAC) thermodilution CO (COtd) and continuous CO (CCO) are pooled to be one variable (Z-statistic). Solid line, mean difference (bias); dotted line, limits of agreement (bias $\pm 1.96 \mathrm{SD}$ ). 

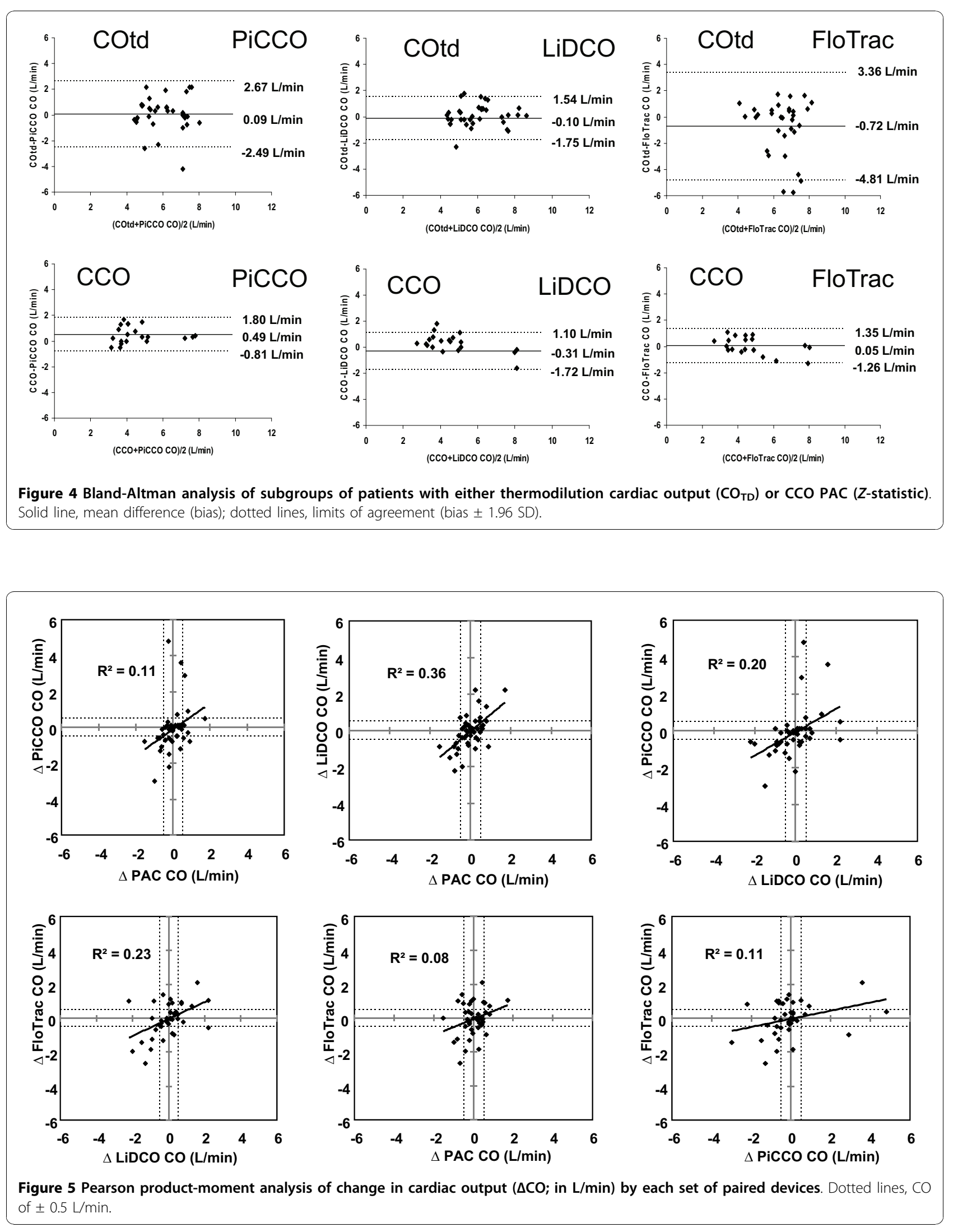
(Figure 3). Second, we studied three separate types of resuscitation interventions (volume loading, vasoactive drug use and inotropic agent use) which reflect clinically relevant scenarios. To date, all published validation studies cited above examined only the ability of these devices to track cardiac output changes in response to volume loading when vasoactive drug therapy was held constant. Although changes in $\mathrm{CO}$ in response to volume loading are very important to document, the impact of other vasoactive therapies are equally important, commonly seen in the clinical setting and potentially confounding to the accuracy of pulse pressurederived estimates of $\mathrm{CO}$.

In support of our findings, recent studies with FloTrac $^{\text {Tw }}$ showed limited accuracy compared to PAC [18,19,31]. Mayer et al. [31] showed in intraoperative cardiac surgery patients that FloTrac ${ }^{\mathrm{Tm}}$ displayed an overall percentage error of $46 \%$ compared to paired COtd values. Potentially, these previous studies unfairly studied FloTrac ${ }^{\mathrm{Tu}}$ by using profound vasomotor paralysis and flow labile states, a clinical limitation specifically cautioned by the manufacturer. Our FloTrac ${ }^{\mathrm{mm}}$ device was equipped with the second-generation software modified to be more accurate in labile states. However, Compton et al. [33] reported continued poor limits of agreement between this second-generation FloTrac ${ }^{\mathrm{mu}}$ algorithm and PiCCO plus ${ }^{\mathrm{Tm}}$ thermodilution $\mathrm{CO}$ measures. Thus, our FloTrac ${ }^{\text {su }}$-PAC data agree with their findings. FloTrac ${ }^{\mathrm{Tx}}$ has subsequently developed a thirdgeneration software algorithm that we did not use. We do not know if this newer iteration will improve Flo$\operatorname{Trac}^{\mathrm{Tm}}$ accuracy, since that modification allowed Flo$\mathrm{Trac}^{\mathrm{Tm}} \mathrm{CO}$ estimates to remain accurate during decoupling states, such as sepsis, which were conditions not present in our cohort. Conversely, PiCCO plus ${ }^{\mathrm{mm}}$ calibration appears to remain accurate within $6 \mathrm{~h}$ of calibration even when vascular tone has been changed [34].

We had nearly equal numbers of patients studied with $\mathrm{CO}_{\mathrm{TD}}$ and CCO PAC. This allowed us to compare these measures with pulse contour analysis. Since both $\mathrm{CO}_{\mathrm{TD}}$ and $\mathrm{CCO}$ are clinically acceptable as part of standard of care in the ICU, this distribution of patients makes our data more robust as a reference for standard ICU care. Regrettably, both FloTrac ${ }^{\mathrm{Tm}}$ and LiDCO Plus ${ }^{\mathrm{mt}} \mathrm{CO}$ values had poor bias and precision with $\mathrm{PAC}$-derived $\mathrm{CO}$ values for both COtd and $\mathrm{CCO}$. These findings are also consistent with the findings of others [18,19,35-37]. Since we did not compare COtd to CCO in the same patient because of the observational nature of our study, we cannot comment on the potential bias between COtd and CCO. However, independently of which PAC method was used for these comparisons, neither gives actual instantaneous measures of CO. COtd measures require the averaging of three to five separate measures taken over a 5-min interval. If cardiac output is systematically changing during this interval (that is, either increasing or decreasing from the start to the end of the series of thermodilution measures), the calculated $\mathrm{CO}$ value may not reflect instantaneous $\mathrm{CO}$ values taken at the same time. Similarly, CCO uses a moving average algorithm that examines thermal dilution of $3 \mathrm{~min}$, making it highly insensitive to rapid changes in $\mathrm{CO}$. However, in our study, we were concerned only with defining the data collection times as those following specific therapeutic interventions when hemodynamic measures, including heart rate, $\mathrm{CO}$ and mean arterial pressure, were constant. Although such statements of stability are relative considering the unstable nature of the postoperative cardiac surgery patient, for the purposes of $\mathrm{CO}$ measures they were stable over the $5 \mathrm{~min}$ of data collection.

Since absolute $\mathrm{CO}$ measures become increasingly more important at low $\mathrm{CO}$ values $[38,39]$, we assessed agreement among our monitoring devices by post hoc analysis of all measured $\mathrm{CO}$ values $\leq 5 \mathrm{~L} / \mathrm{min}$. We found that the degree of bias decreased slightly relative to the complete $\mathrm{CO}$ data set, although the degree of variability among the devices remained (Figure 2). Accordingly, LiDCO Plus ${ }^{\mathrm{Tw}}$, PiCCO Plus ${ }^{\mathrm{Tm}}$ and FloTrac ${ }^{\mathrm{Tm}}$ cannot be assumed to be interchangeable with PAC devices in the assessment of low $\mathrm{CO}$ values. Again, which device, if any, reports the most accurate value and trend during low flow states is not known on the basis of our study. Furthermore, most of the variance between $\mathrm{LiDCO}^{\mathrm{tw}}$ and FloTrac $^{\text {Tx }}$ with PAC-derived CO measures came from the $\mathrm{CO}_{\mathrm{TD}}$ values, and then when these cardiac output values were $>5 \mathrm{~L} / \mathrm{min}$. This finding is the opposite of what Opdam et al. found [18]. Potentially, averaging CO measurements over $20 \mathrm{~s}$ improved agreement between the devices and $\mathrm{CCO}$ as opposed to those and $\mathrm{CO}_{\mathrm{TD}}$ PAC. This difference between $\mathrm{CCO}$ and COtd may reflect the clinical decision bias by which patients with intrinsically lower $\mathrm{CO}$ get $\mathrm{CCO}$ devices $(4.8 \pm 1.4 \mathrm{l}$ / $\min$ ), whereas those with high $\mathrm{CO}$ get $\mathrm{CO}_{\mathrm{TD}}$ devices $(6.0 \pm 1.3 \mathrm{l} / \mathrm{min})$.

One major potential benefit of using $\mathrm{CCO}$ monitoring is to note directional changes in flow. By Pearson product-moment analysis, we found poor correlation between each device pair, with the best correlation between LiDCO Plus ${ }^{\mathrm{Tm}}$ and FloTrac ${ }^{\mathrm{Tm}}$. PiCCO Plus ${ }^{\mathrm{Th}}$ Pearson product-moment analysis accuracy was intermediate between LiDCO $^{\text {tw }}$ and FloTrac ${ }^{\text {tm }}$.

That these devices differed in their paired performances is not surprising. They all use different aspects of the arterial pulse and rely on different assumptions in their $\mathrm{CO}$ estimations. Most of our patient cohort was being administered varying levels of vasoactive 
medications that must alter their vasomotor tone at baseline and over time. Since LiDCO Plus ${ }^{\mathrm{TM}}$ and Flo$\operatorname{Trac}^{\mathrm{TN}}$ use similar aspects of the arterial pulse to calculate $\mathrm{CO}$, this may explain their better concordance by Pearson product-moment analysis. Also, volume challenge in preload-responsive patients increases $\mathrm{CO}$ by $>$ $10 \%-15 \%[33,40]$. We used this threshold $\mathrm{CO}$ value as a minimal $\mathrm{CO}$ change and still observed poor agreement between devices.

\section{Study limitations}

First, we report on a small patient cohort, limiting subgroup analysis and potentially showing differences when a larger number of patients would show similarity. Not all patients received all therapies, since our study was observational. Still, this limitation reflects real-life conditions. Yet, patients are treated individually, not as group means, thus these data are relevant to clinical decision making. Second, we did not use the $\mathrm{PiCCO}^{\mathrm{im}}$ or $\mathrm{LiDCO}^{\mathrm{mm}}$ device-specific calibration methods. However, our common baseline external calibration method is approved by both manufacturers as an acceptable method. Since our goal was to ascertain the dynamic accuracy of these devices, we reasoned that starting from a common $\mathrm{CO}$ value using an external calibration method would maximize potential $\mathrm{CO}$ agreements between devices. If anything, separate $\mathrm{PiCCO}^{\text {ma }}$ and $\mathrm{LiDCO}^{\mathrm{ma}}$ calibrations would produce more, not less, $\mathrm{CO}$ variance than we report. Third, we compared not only mean $\mathrm{CO}$ values but also their changes and Pearson product-moment analysis as recommended by Squara et al. [21]. They also recommended assessment of dynamic real-time trends as a fourth method of analysis. We did not use this fourth method of comparison, because COtd did not lend itself to it. Finally, not all of our patients had femoral arterial catheters, which might have affected the result of $\mathrm{PiCCO}^{\mathrm{ma}} \mathrm{CO}$ estimates, as large peripheral arteries are their preferred sites. However, the femoral (central arterial) site requirement is such that the thermal calibration signal can pass the sensing thermistor not for subsequent $\mathrm{CO}$ estimates. The manufacturer allows for radial site insertion with external calibration. Furthermore, we saw no systematic differences in agreement from femoral and radial site $\mathrm{PiCCO} \mathrm{CO}$ measures. Thus, the PiCCO data reflect the accurate values.

\section{Conclusions}

LiDCO Plus ${ }^{\mathrm{mm}}$, PiCCO $^{\mathrm{mm}}$, FloTrac ${ }^{\mathrm{Tm}}$ and PAC did not show similar $\mathrm{CO}$ trending results, although all produced similar pooled steady-state $\mathrm{CO}$ values. Furthermore, if clinical trials of resuscitation based on $\mathrm{CO}$ values show efficacy when using one of these devices, it is not clear whether performing the identical trial with another $\mathrm{CO}$ monitoring device will also show similar benefit. Thus, until the agreement among minimally invasive $\mathrm{CO}$ measuring devices improves, each device needs to have its own clinical efficacy validated.

\section{Key messages}

- Since the PAC-derived estimates of cardiac output by the thermodilution technique are not the gold standard for estimating cardiac output at the bedside, all available measures of cardiac output need to be compared to each other rather than to a PAC reference.

- Different commercially available arterial pressurederived estimates of cardiac output give differing degrees of error relative to each other.

- The cardiac output error among devices is low for cardiac output values $<5 \mathrm{~L} / \mathrm{min}$.

- Studies documenting clinical benefit using catheter-derived estimates of cardiac output to drive resuscitation algorithms using one monitoring device cannot be extrapolated to similar utility by using another cardiac output monitoring device.

\section{Abbreviations}

CCO: cardiac output by continuous technique; CO: cardiac output; COtd: cardiac output by thermodilution technique; $\mathrm{DO}_{2}$ : oxygen delivery; ICU: intensive care unit; PAC: pulmonary artery catheter.

\section{Competing interests}

MRP is a member of the medical advisory boards for and has received honoraria for lectures from both LiDCO Ltd and Edwards LifeSciences, Inc, and has stock options with LiDCO Ltd. All other authors declare that they have no competing interests.

\section{Authors' contributions}

$\mathrm{MH}$ helped design the study, recruited the patients, collected the data, analyzed the initial data and wrote the first draft of the manuscript. HKK helped analyze the data and edited the later versions of the manuscript. DS helped collect and store the data and performed the preliminary statistical analysis. MRP helped design the study, got Institutional Review Board approval, analyzed the data and wrote all versions of the manuscript.

\section{Acknowledgements}

The authors thank the Cardiothoracic Intensive Care Unit nursing staff at Presbyterian University Hospital, University of Pittsburgh Medical Center, for their support in conducting the study. Also, we appreciate both Edwards LifeSciences and LiDCO companies for providing us with the devices, supplies and training for the study. This work was supported in part by National Institutes of Health grants HL67181 and HL073198.

\section{Author details}

'Department of Critical Care Medicine, University of Pittsburgh Medical Center, 230 Lothrop Street, Pittsburgh, PA 15261, USA. ${ }^{2}$ Cardiothoracic Surgery, University of Pittsburgh Medical Center, 230 Lothrop Street, Pittsburgh, PA 15261, USA. ${ }^{3}$ Current address: Eisenhower Medical Center, 39000 Bob Hope Drive, Rancho Mirage, CA 92270, USA.

\section{Received: 5 May 2010 Revised: 8 September 2010}

Accepted: 23 November 2010 Published: 23 November 2010

\section{References}

1. Swan HJ, Ganz W, Forrester J, Marcus H, Diamond G, Chonette D: Catheterization of the heart in man with use of a flow-directed balloontipped catheter. N Engl J Med 1970, 283:447-451. 
2. Gomez CM, Palazzo MG: Pulmonary artery catheterization in anaesthesia and intensive care. Br J Anaesth 1998, 81:945-956.

3. Rapoport J, Teres D, Steingrub J, Higgins T, McGee W, Lemeshow S: Patient characteristics and ICU organizational factors that influence frequency of pulmonary artery catheterization. JAMA 2000, 283:2559-2567.

4. Connors AF Jr, Speroff T, Dawson NV, Thomas C, Harrell FE Jr, Wagner D, Desbiens N, Goldman L, Wu AW, Califf RM, Fulkerson WJ, Vidaillet H, Broste S, Bellamy P, Lynn J, Knaus WA, the SUPPORT Investigators: The effectiveness of right heart catheterization in the initial care of critically ill patients. JAMA 1996, 276:889-897.

5. Sandham JD, Hull RD, Brant RF, Knox L, Pineo GF, Doig CJ, Laporta DP, Viner S, Passerini L, Devitt H, Kirby A, Jacka M, the Canadian Critical Care Trials Group: A randomized, controlled trial of the use of pulmonaryartery catheters in high-risk surgical patients. N Engl J Med 2003, 348:5-14.

6. Harvey $S$, Harrison DA, Singer $M$, Ashcroft J, Jones C, Elbourne D, Brampton W, Williams D, Young D, Rowan K: Assessment of the clinical effectiveness of pulmonary artery catheters in management of patients in intensive care (PAC-Man): a randomized controlled trial. Lancet 2005, 366:472-477

7. National Heart and Blood Institute Acute Respiratory Distress Syndrome (ARDS) Clinical Trials Network, Wheeler AP, Bernard GR, Thompson BT, Schoenfeld D, Wiedemann HP, deBoisblanc B, Connors AF Jr, Hite RD, Harabin AL: Pulmonary-artery versus central venous catheter to guide treatment of acute lung injury. N Engl J Med 2006, 354:2213-2224.

8. Hadian M, Pinsky MR: Evidence based of the use of the pulmonary artery catheter: impact data and complications. Crit Care 2006, 10(Suppl 3): S11-S18.

9. Wesseling $\mathrm{KH}$, de Witt B, Weber JA: A simple device for continuous measurement of cardiac output. Adv Cardiovasc Phys 1983, 5:16-52.

10. Tannenbaum GA, Mathews D, Weissman C: Pulse contour cardiac output in surgical intensive care unit patients. J Clin Anesth 1993, 5:471-478.

11. Godje O, Hoke K, Goetz AE, Felbinger TW, Reuter DA, Reichart B, Friedl R, Hannekum A, Pfeiffer UJ: Reliability of a new algorithm for continuous cardiac output determination by pulse-contour analysis during hemodynamic instability. Crit Care Med 2002, 30:52-58.

12. Headley JM: Arterial pressure-based technologies: a new trend in cardiac output monitoring. Crit Care Nurs Clin North Am 2006, 18:179-187.

13. Hamilton $\Pi$, Huber $L M$, Jessen ME: PulseCO: a less-invasive method to monitor cardiac output from arterial pressure after cardiac surgery. Ann Thorac Surg 2002, 74:S1408-S1412.

14. Pittman J, Bar-Yosef S, SumPing J, Sherwood M, Mark J: Continuous cardiac output monitoring with pulse contour analysis: a comparison with lithium indicator dilution cardiac output measurement. Crit Care Med 2005, 33:2015-2021.

15. Felbinger TW, Reuter DA, Eltzschig HK, Bayerlein J, Goetz A: Cardiac index measurement during rapid preload changes: a comparison of pulmonary artery thermodilution with arterial pulse contour analysis. $J$ Clin Anesth 2005, 17:241-248

16. Manecke GR: Edwards FloTrac ${ }^{\mathrm{TM}}$ sensor and Vigileo ${ }^{\mathrm{TM}}$ monitor: easy, accurate, reliable cardiac output assessment using the arterial pulse wave. Expert Rev Med Devices 2005, 2:523-527.

17. McGee W, Horswell $J$, Calderon J, Janvier G, Van Severen T, Van den Berge G, Kozikowski L: Validation of a continuous, arterial pressure-based cardiac output measurement: a multicenter, prospective clinical trial. Crit Care 2007, 11:R105.

18. Opdam HI, Wan L, Bellomo R: A pilot assessment of the FloTrac ${ }^{\mathrm{TM}}$ cardiac output monitoring system. Intensive Care Med 2007, 33:344-349.

19. Mayer J, Boldt J, Schollhorn T, Röhm KD, Mengistu AM, Suttner S: Semiinvasive monitoring of cardiac output by a new device using arterial pressure waveform analysis: a comparison with intermittent pulmonary artery thermodilution in patients undergoing cardiac surgery. $\mathrm{Br} J$ Anaesth 2007, 98:176-182.

20. Biancofiore G, Critchley LA, Lee A, Bindi L, Bisà M, Esposito M, Meacci L, Mozzo R, DeSimone P, Urbani L, Filipponi F: Evaluation of an uncalibrated arterial pulse contour cardiac output monitoring system in cirrhotic patients undergoing liver surgery. $\mathrm{Br} J$ Anaesth 2009, 102:47-54.

21. Squara $P$, Cecconi $M$, Rhodes $A$, Singer M, Chiche JD: Tracking changes in cardiac output: methodological considerations for the validation of monitoring devices. Intensive Care Med 2009, 35:1801-1808.
22. Pearse R, Dawson D, Fawcett J, Rhodes A, Grounds RM, Bennett ED: Early goal-directed therapy after major surgery reduces complications and duration of hospital stay [ISRCTN38797445]. Crit Care 2005, 9:R687-R693.

23. Pratt B, Roteliuk L, Hatib F, Frazier J, Wallen RD: Calculating arterial pressure-based cardiac output using a novel measurement and analysis method. Biomed Instrum Technol 2007, 41:403-411.

24. WINDAQ Acquisition and Playback Software In-Depth Presentation: 2010 [http://www.dataq.com/applicat/index.htm], Accessed November 24.

25. Bland JM, Altman DG: Statistical method for assessing agreement between two methods of clinical measurements. Lancet 1986, 1:307-310.

26. Critchley LAH, Critchley JAJH: A meta-analysis of studies using bias and precision statistics to compare cardiac output measurement techniques. J Clin Monit Comput 1999, 15:85-91.

27. Pearse R, Dawson D, Fawcett J, Rhodes A, Grounds RM, Bennett ED: Early goal-directed therapy after major surgery reduces complications and duration of hospital stay: a randomized, controlled trial [ISRCTN38797445]. Crit Care 2005, 9:R687-R693.

28. McKendry M, McGloin $H$, Saberi $D$, Caudwell $L$, Brady AR, Singer M: Randomised controlled trial assessing the impact of a nurse delivered, flow monitored protocol for optimisation of circulatory status after cardiac surgery. BMJ 2004, 329:256.

29. Mayer J, Boldt J, Mengistu AM, Röhm KD, Suttner S: Goal-directed intraoperative therapy based on autocalibrated arterial pressure waveform analysis reduces hospital stay in high-risk surgical patients: a randomized, controlled trial. Crit Care 2010, 14:R18.

30. Pölönen $\mathrm{P}$, Ruokonen $\mathrm{E}$, Hippeläinen $\mathrm{M}$, Pöyhönen $\mathrm{M}$, Takala J: A prospective, randomized study of goal-oriented hemodynamic therapy in cardiac surgical patients. Anesth Analg 2000, 90:1052-1059.

31. Mayer J, Boldt J, Schollhorn T, Rohm KD, Mengistu AM, Suttner S: Semiinvasive monitoring of cardiac output by a new device using arterial pressure waveform analysis: a comparison with intermittent pulmonary artery thermodilution in patients undergoing cardiac surgery. $\mathrm{Br} J$ Anaesth 2007, 98:176-182.

32. Marquez J, McCurry K, Severyn DA, Pinsky MR: Ability of pulse power, esophageal Doppler and arterial pressure to estimate rapid changes in stroke volume in humans. Crit Care Med 2008, 36:3001-3007.

33. Osman D, Ridel C, Ray P, Monnet X, Anguel N, Richard C, Teboul JL: Cardiac filling pressures are not appropriate to predict hemodynamic response to volume challenge. Crit Care Med 2007, 35:64-68.

34. Hamzaoui O, Monnet X, Richard C, Osman D, Chelma D, Teboul JL: Effects of changes in vascular tone on the agreement between pulse contour and transpulmonary thermodilution cardiac output measurements within an up to 6-hour calibration-free period. Crit Care Med 2008, 36:434-440.

35. Compton FD, Zukunft B, Hoffmann C, Zidek W, Schaefer JH: Performance of a minimally invasive uncalibrated cardiac output monitoring system $\left(\right.$ FloTrac $^{\mathrm{TM}} /$ Vigileo $^{\mathrm{TM}}$ ) in haemodynamically unstable patients. $\mathrm{Br} J$ Anaesth 2008, 100:451-456.

36. Rodig G, Prasser C, Keyl C, Liebold A, Hobbhalm J: Continuous cardiac output measurement: pulse contour analysis vs. thermodilution technique in cardiac surgical patients. Br J Anaesth 1999, 82:525-530.

37. Yamashita K, Nishiyama T, Yokoyama T, Abe H, Manabe M: Cardiac output by PulseCO ${ }^{\mathrm{TM}}$ is not interchangeable with thermodilution technique in patients undergoing OPCAB. Can J Anaesth 2005, 52:530-534.

38. Pinsky MR, Vincent $J$ : Let us use the pulmonary artery catheter correctly and only when we need it. Crit Care Med 2005, 33:1119-1122.

39. Pinsky MR, Payen D: Functional hemodynamic monitoring. Crit Care 2005, 9:566-572.

40. Monnet X, Rienzo M, Osman D, Anguel N, Richard C, Pinsky MR, Teboul LL: Passive leg raising predicts fluid responsiveness in the critically ill. Crit Care Med 2006, 34:1402-1407.

\section{doi:10.1186/cc9335}

Cite this article as: Hadian et al.: Cross-comparison of cardiac output trending accuracy of LiDCO, PiCCO, FloTrac and pulmonary artery catheters. Critical Care 2010 14:R212. 